\title{
Impactos Socioeconômicos no Brasil do Modelo MPS-SW para Melhoria de Processos de Software
}

\author{
Kival Chaves Weber ${ }^{1}$, Mariano de Matos Macedo ${ }^{2}$, Nelson Henrique Franco de \\ Oliveira $^{3}$, Elidiane Teixeira Barroso ${ }^{3}$ e Virgínia Costa Duarte ${ }^{3}$
}

${ }^{1}$ Consultor Sênior em TIC, Qualidade e Inovação - Rua Carneiro Lobo 153, apt 1402 80240.240 - Curitiba-PR
${ }^{2}$ Professor de Economia na UFPR - Universidade Federal do Paraná - Rua Euclides Bandeira 500, apt 1203 - 80530.020 - Curitiba-PR
${ }^{3}$ Softex - Associação para Promoção da Excelência do Software Brasileiro - Rua Irmã Serafina $863,6^{\circ}$ andar - 13015.914 - Campinas-SP
kival_weber@yahoo.com.br, mariano.macedo@ufpr.br, \{nelson, elidiane, virginia\} @softex.br

Resumo. O programa MPS.BR foi lançado em dezembro de 2003 visando à criação e difusão do modelo MPS-SW. Os resultados alcançados superaram os resultados esperados e foram conduzidas pesquisas visando obter evidências adicionais: de 2008-2013, seis pesquisas anuais com foco na melhoria do desempenho técnico, denominadas 'iMPS - Resultados de Desempenho das Organizações que Adotaram o Modelo MPS-SW'; em 2014, a $1^{a}$ pesquisa de satisfação dos clientes MPS-SW com foco na melhoria do desempenho organizacional, denominada 'MPS quer ouvir você!'. Este artigo apresenta os resultados de outra pesquisa conduzida em 2014-2015 com foco nos impactos socioeconômicos do MPS-SW no Brasil, denominada 'MPS Cidadão'.

\begin{abstract}
In December 2003 it was launched the MPS.BR program aiming at establishing and disseminating the MPS-SW model. The achieved results exceeded the predefined benchmarks and there were conducted surveys aiming at attaining further evidences: from 2008-2013, six annual surveys focused on the technical performance improvement, named 'iMPS - Performance Results of Organizations that Adopted the MPS-SW Model'; in 2014, the $1^{\text {st }} M P S-S W$ customer satisfaction survey focused on the organizational performance enhancement, named 'MPS wants to hear you!'. This paper presents the results of another survey conducted in 2014-2015 focused on the socioeconomic impacts of the MPS-SW model in Brazil, named 'MPS Citizen'.
\end{abstract}

\section{Introdução}

No início dos anos 2000 estudos mostravam que seria necessário um esforço significativo para aumentar a maturidade dos processos de software nas empresas brasileiras pois, nos anos 1990, as empresas de software no Brasil favoreceram a certificação ISO 9000, genérica, em detrimento de outras normas e modelos especificamente voltadas para a melhoria de processos de software [MCT/SEPIN 2001, Veloso et al 2003].

Isto motivou o lançamento em 11Dez2003 do programa MPS.BR - Melhoria de Processo do Software Brasileiro, sob coordenação da Softex, com duas metas 
desafiadoras: i) uma meta técnica visando à criação e ao aprimoramento do modelo MPS$\mathrm{SW}$; ii) uma meta de negócio visando à difusão deste modelo em todas as regiões do país [Weber et al 2004].

Em 2004 foi criado o modelo de negócio MN-MPS, com regras de negócio vigentes até hoje. No SBQS 2005 foram lançados o modelo de referência MR-MPS e o método de avaliação MA-MPS [Weber et al 2005]. Face aos resultados alcançados de 2004 a 2008, o MPS.BR começou a ser reconhecido no país e no exterior como um programa bem sucedido [Montoni et al 2009]. No SBES 2011 foi destacada a contribuição do modelo MPS-SW para as pesquisas brasileiras na área de engenharia de software [Kalinowski et al 2011]. No QUATIC 2012, em Lisboa, o programa MPS.BR foi apresentado como um caso de sucesso no Brasil da Tripla Hélice (Academia, Governo e Indústria) [Etzkowitz and Mello 2004, Santos et al 2012]. Em 2014 foi publicado o 'Estudo de caso: 10 anos de MPS.BR' [Weber et al 2014a].

Este artigo apresenta os resultados finais da pesquisa 'MPS Cidadão', realizada de Nov2014-Mai2015. A seção 2 trata tanto do programa MPS.BR e do modelo MPS, quanto dos resultados de outras pesquisas MPS: de 2008-2013, a série de pesquisas anuais iMPS com foco na melhoria do desempenho técnico das organizações; em 2014, a $1^{\text {a }}$ pesquisa de satisfação dos clientes MPS-SW com foco na melhoria do desempenho organizacional, denominada 'MPS quer ouvir você!'. Na seção 3 são apresentados objetivo, método, caracterização e resultados das duas partes da pesquisa 'MPS Cidadão' com foco nos impactos sociais e econômicos do modelo MPS-SW no Brasil. Na seção 4 são apresentadas as conclusões do artigo e as considerações finais.

\section{Programa MPS.BR e Modelo MPS}

A fundamentação teórica da melhoria de processos de software e do modelo MPS-SW pode ser encontrada nas referências deste artigo e também no Guia Geral de Software: 2012 que detalha o Modelo de Referência MR-MPS-SW, com sete níveis de maturidade no qual o mais baixo é o nível G é o mais alto é o nível A, baseado tanto na ISO/IEC 12207 - Processos do Ciclo de Vida do Software, quanto no CMMI-DEV, e no Guia de Avaliação: 2013 que descreve o Processo e o Método de Avaliação MA-MPS, baseado na ISO/IEC 15504 - Avaliação de Processo (ver http://www.softex.br/mpsbr/guias/).

Até 2014, os resultados alcançados no programa MPS.BR e no modelo MPS-SW superaram os resultados esperados. Desde 2004, mais de 6.000 pessoas participaram de cursos oficiais MPS. De 2005-2014, foram publicadas no sítio Web www.softex.br/mpsbr mais de 600 avaliações MPS-SW realizadas em todas as cinco regiões brasileiras. De 2011-2014, a adoção do MPS-SW cresceu a uma taxa de 100 avaliações por ano.

Os fatores críticos de sucesso foram:

- A criação e o aprimoramento do modelo MPS-SW pela ETM - Equipe Técnica do Modelo que é composta por membros convidados pela Softex, escolhidos entre profissionais na área (60\% da Academia).

- O inovador modelo de negócio MN-MPS que define as regras de negócio do MPSSW, baseado no Triângulo de Serviços [Teboul 2008]: i) a empresa responsável pelo modelo MPS (Softex); ii) a abrangente rede MPS de colaboradores da linha de frente (instrutores de cursos oficiais MPS, ETM - Equipe Técnica do Modelo MPS, IOGE - Instituições Organizadoras de Grupos de Empresa MPS, II - 
Instituições Implementadoras MPS, IA - Instituições Avaliadoras MPS e ICA Instituições de Consultoria de Aquisição); iii) o cliente MPS.

- A aceleração da difusão do modelo MPS-SW nas 5 regiões do país como resultado do apoio parcial a grupos de empresas por meio de COMUNICADOS Softex, com recursos nacionais do MCTI, FINEP e SEBRAE e recursos internacionais do BID/FUMIN [Bercovitch 2014], em contrapartida a recursos tanto das empresas participantes nos grupos quanto de outras fontes locais. Este modelo de negócio cooperado baseou-se nas lições aprendidas pelos Agentes Regionais Softex na organização de grupos de empresas Rumo à ISO 9000 e ao CMMI.

Visando obter evidências adicionais, foram conduzidas pesquisas sobre dois tipos de impactos do modelo MPS-SW:

- Impacto do MPS-SW na melhoria do desempenho técnico: Em 2008, a Softex contratou o Grupo de Engenharia de Software Experimental da COPPE/UFRJ para modelar o estudo iMPS - Resultados de Desempenho das Organizações que Adotaram o Modelo MPS e realizar anualmente pesquisas iMPS [Kalinowski et al 2008]. De 2008 a 2013, foram conduzidas seis pesquisas anuais iMPS sobre o 'impacto do MPS-SW na melhoria do desempenho técnico' das organizações, com coleta de dados junto aos respectivos Gerentes de Projeto e da Qualidade. $\mathrm{Na}$ pesquisa iMPS2013 foram recebidos 181 questionários de 148 empresas diferentes. A caracterização desta pesquisa reforça que, quanto mais alto o nível de maturidade MPS, melhor o desempenho, a precisão de estimativa e a qualidade. As empresas também mencionaram perceber contribuições do modelo para a inovação de seus processos e, eventualmente, de seus produtos. A satisfação com o modelo MPS-SW permanece alta (maior que 95\%). A análise global das pesquisas iMPS desde 2008, em amostra composta por 292 organizações, permitiu obter indicações mais explícitas sobre os benefícios do MPS-SW para as empresas que o utilizam e evidencia a importância da busca por níveis mais altos de maturidade à medida que o tamanho da empresa e o perfil dos projetos evolui [Travassos e Kalinowski 2014, Kalinowski et al 2014].

- Impacto do MPS-SW na melhoria do desempenho organizacional: A $1^{\text {a }}$ pesquisa de satisfação dos clientes MPS-SW, denominada 'MPS quer ouvir você!', foi conduzida pela Softex com coleta de respostas junto aos patrocinadores de avaliações MPS vigentes (dentro do prazo de validade de três anos) ou não - que normalmente são membros da Alta Direção das organizações. De Fev-Jun2014, foram enviados 403 questionários online SurveyMonkey Gold e recebidas 190 respostas completas (47\%). Quanto à probabilidade do patrocinador da avaliação 'recomendar, para um amigo ou colega, o MPS-SW como um modelo para a melhoria do desempenho organizacional': i) é muito alta (93\% deram nota de 7 a 10) e bem definida para o grande número de respostas de patrocinadores de avaliações MPS-SW vigentes; ii) é mais baixa embora ainda relativamente alta (77\% deram nota de 7 a 10) e difusa para o pequeno número de respostas de patrocinadores que não renovaram suas avaliações MPS-SW [Weber et al 2014b, Kalinowski et al 2014]. 


\section{Pesquisa 'MPS Cidadão'}

Esta seção apresenta as duas partes da pesquisa 'MPS Cidadão' sobre os impactos socioeconômicos do MPS-SW no Brasil, conduzidas pela Softex de Nov2014-Mai2015, resumindo objetivo, método, caracterização e resultados finais.

\subsection{Impactos socioeconômicos do MPS-SW no Brasil}

[Objetivo] A $1^{\text {a }}$ parte da pesquisa 'MPS Cidadão' visa analisar o 'impacto socioeconômico do MPS-SW no Brasil', com base em respostas de patrocinadores de avaliações MPS vigentes.

Emprego e Salário Médio nas
Empresas MPS
- Empregos de boa qualidade
gerados pelas empresas MPS: $\%$
elevado de pessoas com níve
superior.
- Salários médios relativamente
elevados.

Inovação nas Empresas MPS - Taxa de Inovação de produtos e processos nas empresas com avaliação MPS é superior à média do segmento.

Pesquisa e Desenvolvimento - Níveis de dispêndios de P\&D (\% da RLV) relativamente expressivos.

Apoio do Governo ou outras instituições de natureza pública para Atividades Inovativas

- Empresas com avaliação MPSSW, com qualidade de processos assegurada, tendem a aumentar a eficácia do apoio (recursos não reembolsáveis, incentivos fiscais, bolsas, etc)

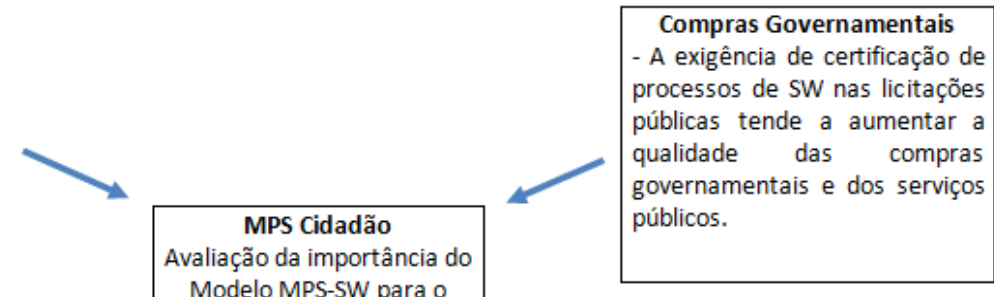
MPS-SW para cidadão e a sociedade. Nova pesquisa complementar à iMPS e à $\quad$ Direcionamento das Vendas ‘MPS Quer Ouvir Você!' Interno

- O uso de SW e serviços é pervasivo a diversas atividades produtivas.

Inserção Global das Empresas MPS Parcerias internacionais

- Expressividade das exportações de SW e serviços
- A aquisição de SW é a $2^{\text {a }}$ mais importante atividade inovativa das empresas, inferior somente à aquisição de máquinas e equipamentos.

Quanto mais difuso forem os segmentos de atividade econômica para os quais as Empresas MPS vendem seus

SW e serviços, maior a contribuição do Modelo MPSSW para a sociedade.

Figura 1. $1^{\text {a }}$ parte da pesquisa 'MPS Cidadão': Marco analítico com variáveis de análise para avaliação dos impactos socioeconômicos do MPS-SW no Brasil

[Método] Foi usada a ferramenta SurveyMonkey Gold < pt.surveymonkey.com > para criar questionário, coletar respostas e analisar resultados. O marco analítico da $1^{\mathrm{a}}$ parte da pesquisa é mostrado na Figura 1. Nas empresas que adotaram o MPS, quanto mais positivos os aspectos relacionados às variáveis selecionadas, maior a importância do modelo para a sociedade e o cidadão. Essas variáveis são as seguintes: empregos de nível superior e salário médio; inovação de produtos ou processos; dispêndios de Pesquisa e Desenvolvimento (P\&D); apoio do governo ou outras instituições de natureza pública para atividades inovativas (recursos não reembolsáveis, SEBRAETEC, incentivos fiscais, bolsas, etc); compras governamentais; inserção global das empresas; e direcionamento das vendas no mercado interno. No que se refere aos conceitos de inovação de produtos ou processos e de $\mathrm{P} \& \mathrm{D}$, a pesquisa 'MPS Cidadão' segue os estabelecidos pela Pesquisa de Inovação (PINTEC) [IBGE 2011] que, por sua vez, recorre aos especificados no 
Manual de Oslo [OECD 2005]. Esses conceitos estão plenamente especificados no questionário da pesquisa.

[Caracterização] De Nov2014-Mai2015, a Softex enviou questionários online para 252 patrocinadores de avaliações MPS-SW vigentes e recebeu 123 respostas (49\%). Quanto à localização geográfica das organizações que responderam: Sudeste $=48 \%$, Sul $=33 \%$, Nordeste $=13 \%$, Norte $=3 \%$ e Centro-Oeste $=3 \%$. Quanto à atividade econômica principal da empresa, predomina o Desenvolvimento de Software e outros Serviços de TI (98\%). Quanto à Receita Operacional Bruta (ROB) anual, segundo critério do BNDES: microempresa (até $\mathrm{R} \$ 2,4$ milhões) $=41 \%$, pequena empresa (entre $\mathrm{R} \$ 2,4$ e 16 milhões) $=40 \%$, média empresa (entre R $\$ 16$ e 90 milhões) $=13 \%$, média-grande empresa (entre $\mathrm{R} \$ 90$ e 300 milhões) $=3 \%$ e grande empresa (superior a $\mathrm{R} \$ 300$ milhões) $=3 \%$.

[Resultados] Em 72\% das empresas MPS, mais de 60\% dos empregados são de nível superior. Em 50\% das empresas, o salário médio é superior a 5 salários mínimos. Nesse caso, pode-se dizer que o MPS-SW contribui para criação de empregos de boa qualidade, como os de nível superior e de salários relativamente elevados, o que é bom para a sociedade e o cidadão.

A Taxa de Inovação em produto (software e serviços) das empresas MPS é de $84 \%$. Essa é a proporção das empresas que realizaram inovação em produto no período de referência da pesquisa. No Brasil, segundo a PINTEC [IBGE 2011], a Taxa de Inovação em produto das empresas de serviços de Tecnologia da Informação ${ }^{1}$ é bem menor: $38,0 \%$. A Taxa de Inovação em processo referente a métodos de produção de software ou serviços das empresas MPS é de $89 \%$. No caso de processos referentes a técnicas novas ou significativamente aperfeiçoadas em atividades de apoio à produção nessas empresas (controle da produção, medição de desempenho, controle da qualidade, etc), a Taxa de Inovação é de $81 \%$. No Brasil, segundo a PINTEC, a Taxa de Inovação em processo das empresas de serviços de TI é muito menor: 37,5\%. Assim, é possível afirmar que o MPS-SW está presente em empresas com Taxas de Inovação de produto ou processo expressivamente superiores à média do segmento de referência, o que significa impactos econômicos favoráveis para a sociedade e cidadão brasileiro.

Em 76\% das empresas MPS, das quais mais de 89\% são inovadoras, a importância das atividades inovativas é média ou alta, percentual que, na PINTEC, alcança 56,7\% nas empresas inovadoras em serviços de TI. Segundo a PINTEC, a proporção da Receita Líquida de Vendas (RLV) dispendida em atividades internas de P\&D pelas empresas inovadoras brasileiras em serviços de TI alcançou 1,62\%. No caso das empresas MPS, esse percentual superou de $1,5 \%$ até $3,0 \%$ e mais de $3 \%$ em $18 \%$ e $39 \%$ das empresas, respectivamente. Em geral, uma parcela maior dessas empresas apresenta uma proporção maior da RLV em P\&D do que as empresas inovadoras de serviços de TI.

A proporção das empresas MPS-SW que receberam "apoio do governo ou de outras instituições de natureza pública" é expressiva em todas as formas de apoio, exceto financiamentos da FINEP com recursos reembolsáveis (5\%). Empresas MPS, ou seja com maior qualidade de processos, tendem a aumentar a eficácia desse apoio, o que constitui outra contribuição relevante do MPS-SW para a sociedade ou o cidadão.

\footnotetext{
${ }^{1}$ Essas atividades se referem ao desenvolvimento de software e outros serviços de TI.
} 
Para 33\% das empresas MPS, a importância das compras governamentais é média ou alta. De forma semelhante ao observado no apoio do governo, as empresas MPS tendem a aumentar a qualidade das compras governamentais de software e serviços, o que também constitui uma importante contribuição do MPS para a sociedade ou o cidadão.

A inserção global das empresas MPS é relativamente pequena, embora expressiva em se tratando da média das empresas brasileiras: $11 \%$ desenvolveram software em parceira ou de forma distribuída com empresas ou instituições de outros país; e 7\% dessas empresas auferem mais de 5\% de suas ROB em decorrência de exportações. Pode-se afirmar que, dentro de certos limites, quanto mais expressivas forem as parcerias e o percentual da receita decorrentes de exportações de bens e serviços de maior densidade tecnológica, como software, mais as empresas contribuem como uma variável de excelência para o dinamismo da economia nacional, o que favorece direta ou indiretamente a sociedade e o cidadão brasileiro.

O modelo MPS-SW tem contribuído diretamente no processo de produção dos mais diversos segmentos de atividade econômica e, pela melhoria do desenvolvimento de software que propicia, é relevante como contribuição para a sociedade e o cidadão. Além disso, segundo a PINTEC, a aquisição de software por parte das empresas inovadoras brasileiras (industriais e de serviços, em geral) é a $2^{\mathrm{a}}$ mais importante atividade inovativa dessas empresas, inferior somente à aquisição de máquinas e equipamentos.

Dentre os segmentos que as empresas MPS são fornecedoras relevantes, merecem destaque o Comércio atacadista ou varejista (27\%), a Administração pública, defesa e seguridade social (27\%), Saúde humana e serviços sociais (16\%), Educação (16\%) e Atividades financeiras, de seguros e serviços relacionados (14\%).

\subsection{Contribuições do MPS-SW para o cidadão, a sociedade brasileira, o desenvolvimento regional e o desenvolvimento nacional}

[Objetivo] A $2^{\mathrm{a}}$ parte da pesquisa 'MPS Cidadão' visa identificar contribuições do MPSSW para o cidadão e a sociedade brasileira, o desenvolvimento regional e nacional, com coleta de respostas junto à rede MPS de colaboradores da linha de frente (instrutores, ETM, IOGE, II, IA e ICA). Esta rede abrangente, em si, é uma contribuição relevante do programa MPS.BR e do modelo MPS-SW para o Brasil.

[Método] Usou-se a ferramenta SurveyMonkey Gold para criar questionários, coletar respostas e analisar resultados. Solicitou-se a cada membro da rede até três contribuições, julgadas relevantes e decorrentes da adoção do MPS-SW, para cada um dos quatro quesitos (cidadão e sociedade brasileira, desenvolvimento regional e nacional), com perguntas de resposta aberta. Os resultados foram obtidos por análise de texto.

[Caracterização] De Nov2014-Mai2015, foram recebidas respostas de: 22 dos 37 instrutores (59\%); 11 dos 12 coordenadores da ETM e das suas áreas (92\%); 9 coordenadores das 15 IOGE apoiadas com recursos financeiros da Softex (60\%); 12 coordenadores das 17 II ativas (71\%); 10 coordenadores das 13 IA ativas (77\%); e 2 coordenadores das 2 ICA ativas $(100 \%)$.

[Resultados] A análise de texto das respostas dos membros da rede mostrou que as três palavras mais exibidas: i) nas 99 respostas relativas ao cidadão foram Melhoria (37\%), Software (26\%) e Qualidade (24\%); ii) nas 97 respostas relativas à sociedade 
brasileira foram Software (29\%), Qualidade (22\%) e Empresas (18\%); iii) nas 102 respostas relativas ao desenvolvimento regional foram Software (28\%), Qualidade (19\%) e Competitividade (16\%); iv) nas 101 respostas relativas ao desenvolvimento nacional foram Qualidade (21\%), Melhoria (18\%) e Competitividade (18\%). A Figura 2 exibe a nuvem do acumulado de 399 respostas da rede, na qual as três palavras mais frequentes foram Software (30\%), Empresas (30\%) e Qualidade (22\%).

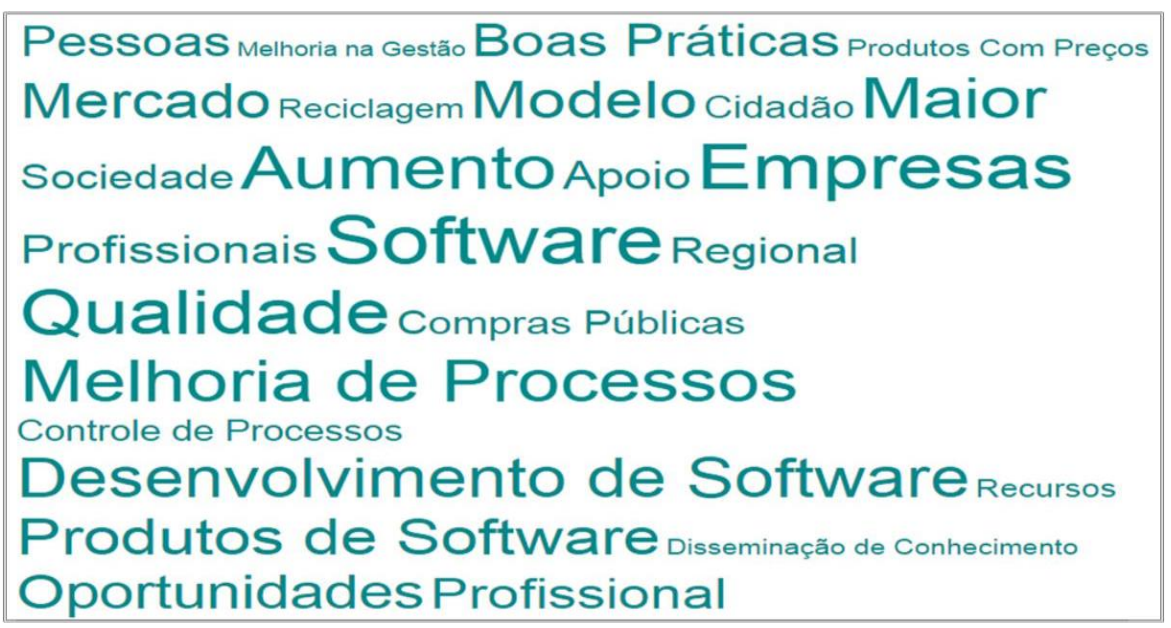

Figura 2. $2^{\mathrm{a}}$ parte da pesquisa 'MPS Cidadão': Exibição em nuvem das contribuições do MPS-SW para o cidadão, a sociedade e o desenvolvimento

\section{Conclusão}

Neste artigo apresentamos objetivo, método, caracterização e resultados finais das duas partes da pesquisa 'MPS Cidadão', conduzidas pela Softex de Nov2014-Mai2015.

Quanto aos impactos socioeconômicos do MPS-SW no Brasil, a $1^{\text {a }}$ parte da pesquisa mostra que os resultados são favoráveis para todas as variáveis selecionadas para análise (empregos de nível superior e salário médio, inovação, dispêndios de P\&D, apoio do governo, etc), o que indica contribuições expressivas deste modelo do ponto de vista dos patrocinadores de avaliações vigentes.

Quanto às contribuições do MPS-SW para o cidadão, a sociedade e o desenvolvimento, a frase "Qualidade de Software nas Empresas" resume o ponto de vista da rede MPS de colaboradores da linha de frente na $2^{\mathrm{a}}$ parte da pesquisa.

Por último, mas não o menos importante, cabe observar que esse esforço sistemático de pesquisas que a Softex vem realizando, tanto sobre a melhoria do desempenho técnico e organizacional quanto sobre os impactos econômicos para a sociedade e o cidadão, não é usual em iniciativas semelhantes. Na realidade, constitui uma busca contínua de evidências objetivas da utilidade do modelo MPS-SW para a melhoria dos processos de software no Brasil, especialmente nas micro, pequenas e médias empresas (MPMEs).

\section{Agradecimentos}

In memoriam. Agradecemos ao Jorge Luis Boria, falecido em 4 de março de 2015, pelas lições ensinadas sobre 'SPI - Software Process Improvement'. Co-autor do livro 'A História da Tahini-Tahini: Melhoria de Processos de Software com Métodos Ágeis e Modelo MPS' (MCTI/SEPIN 2013), mentor e amigo de todos na comunidade de prática do MPS, foi um renomado MPS Senior Advisor, Certified MPS-SW Instructor, 
Certified MPS Competent Assessor, SEI Visiting Scientist, Certified CMMI Instructor, Certified High Maturity SCAMPI Lead Appraiser, Certified Scrum Alliance Scrum Master.

\section{Referências}

Bercovitch, N. Apoyo a la Competitividad Internacional de las Pymes de Software - Un programa para el mejoramiento de la calidad: MPS.BR. Caso $\mathrm{n}^{\circ} 4$ del libro 'Lecciones aprendidas sobre adopción de TIC en PYMES: La experiencia del FOMIN/BID en América Latina. Publicación Fundación Telefonica FOMIN, 2014. http://www.fomin.org/pymespracticas

Etzkowitz, H., Mello, J. The rise of a triple helix culture: innovation in Brazilian economic and social development. International Journal of Technology Management \& Sustainable Development. 2004.

IBGE. Pesquisa de Inovação (PINTEC), 2011. http://www.pintec.ibge.gov.br/

Kalinowski, M., Weber, K., Travassos, G. H. iMPS: an experimentation based investigation of a nationwide software development reference model. In: 2nd International Symposium on Empirical Software Engineering and Measurement (ESEM), Kaiserslautern, Germany, 2008.

Kalinowski, M., Santos, G., Prikladnicki, R., Rocha, A. R., Weber, K., Antonioni, J. A. From software engineering research to Brazilian software quality improvement. In: 25th Brazilian Symposium on Software Engineering, SBES 2011, São Paulo, 2011.

Kalinowski, M., Weber, K., Franco, N., Barroso, E., Duarte, V., Zanetti, D., Santos, G. Results of 10 Years of Software Process Improvement in Brazil Based on the MPS-SW Model. In: 9th Int. Conf. on the Quality in Information and Communications Technology (QUATIC), Guimarães, Portugal, 2014.

MCT/SEPIN. Qualidade e Produtividade no Setor de Software Brasileiro 2001. Brasília: 2001.

Montoni, M. A., Rocha, A. R., Weber, K. MPS.BR: A successful program for software process improvement in Brazil. Software Process: Improvement and Practice, Vol. 14, Issue 5, pp. 289-300, 2009.

OECD. Oslo Manual: Guidelines for Collecting and Interpreting Innovation Data, 3rd Edition, 2005. http://www.oecd-ilibrary.org/science-and-technology/oslo-manual_9789264013100-en

Santos, G., Kalinowski, M., Rocha, A. R, Travassos, G. H., Weber K., Antonioni, J. A. MPS.BR program and MPS model: main results, benefits and beneficiaries of software process improvement in Brazil. In: 8th Int. Conf. on the Quality in Information and Communications Technology (QUATIC), Lisbon, Portugal, 2012.

Teboul, J. Serviços em cena: o diferencial que agrega valor ao seu negócio. - Brasília, Brasil: ELSEVIER, INSEAD, CAMPUS, CNI-IEL/NC, 2008.

Travassos, G. H. e Kalinowski, M. iMPS 2013 - Evidências sobre o Desempenho das Empresas que Adotaram o Modelo MPS-SW. Softex, 2014.

Veloso, F., Botelho, A. J., Tschang, A., Amsden, A. Slicing the Knowledge-based Economy in Brazil, China and India: a tale of 3 software industries. Report, MIT - Massachusetts Institute of Technology, Mass, 2003.

Weber, K., Rocha, A. R., Rouiller, A. C., Crespo, A., Alves, A., Ayala, A., Gonçalves, A., Paret, B., Vargas, C., Salviano, C., Machado, C., Scalet, D., Petit, D., Araújo, E., Maldonado, J. C., Oliveira, K., Oliveira, L. C., Girão, M., Amaral, M., Campelo, R., Maciel, T. Uma Estratégia para Melhoria de Processo de Software nas Empresas Brasileiras. In: 5th Conference for Quality in Information and Communications Technology (QUATIC). Porto, Portugal, 2004.

Weber, K., Araújo, E., Machado, C. F., Scalet, D., Salviano, C. F., Rocha, A. R. Modelo de Referência e Método de Avaliação para Melhoria de Processo de Software - versão 1.0 (MR-MPS e MA-MPS). In: Anais do IV Simpósio Brasileiro de Qualidade de Software (SBQS 2005). Porto Alegre - RS: 2005. Prêmio de melhor Artigo Técnico do SBQS 2005.

Weber, K., Franco, N., Duarte, V. Estudo de caso: 10 anos de MPS.BR. Softex, 2014a.

Weber, K., Franco, N., Barroso, E., Duarte, V., Zanetti, D. Pesquisa qualitativa 'MPS quer ouvir você!': Resumo Executivo. Softex, 2014b. 